\title{
Efecto de la poda y la densidad de siembra sobre el rendimiento y calidad de melón Cantaloupe (Cucumis melo L.) cultivado bajo invernadero
}

\section{Effect of pruning and plant density on yield and quality of Cantaloupe melon (Cucumis melo L.) grown under greenhouse conditions}
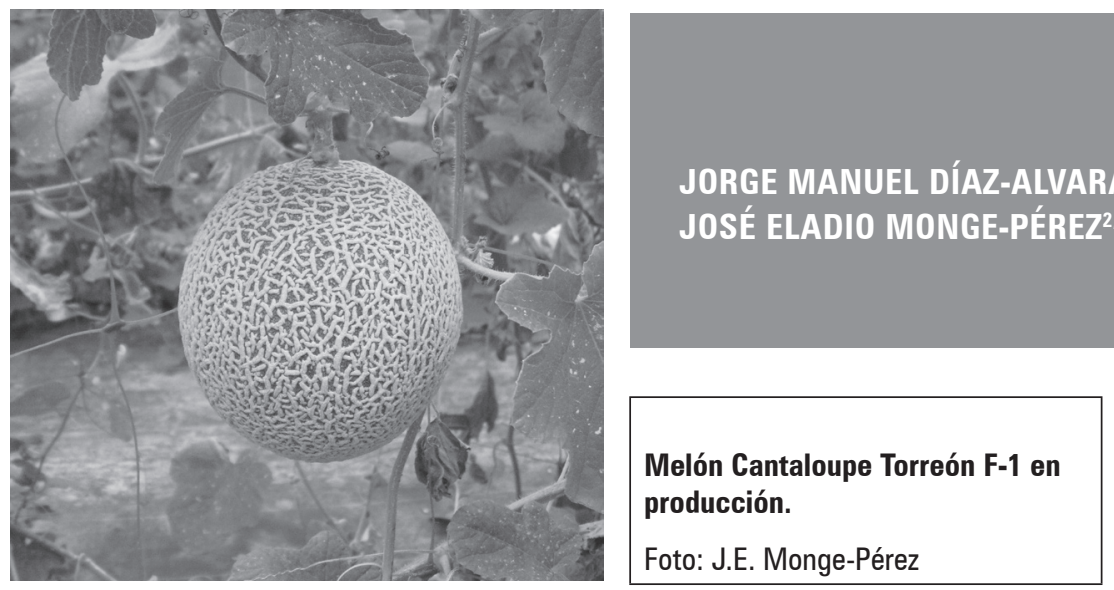

\section{RESUMEN}

Debido a que no existe información sobre el efecto de la poda y la densidad de siembra sobre el cultivo de melón en invernadero en Costa Rica, se planteó el siguiente estudio con el objetivo de evaluar el efecto de tres densidades de siembra (1,9; 3,2 y 3,9 plantas $/ \mathrm{m}^{2}$ ) y tres tipos de poda (un tallo secundario, dos tallos secundarios y plantas sin poda) sobre el rendimiento y la calidad del melón Cantaloupe Torreón F-1. El cultivo se manejó con fertirrigación, en sustrato de fibra de coco. No se presentaron diferencias significativas entre tratamientos para los días a inicio de la cosecha ni para la relación pulpa:cavidad. Se registraron diferencias significativas entre tratamientos, tanto para el rendimiento como para el porcentaje de sólidos solubles totales. El mayor rendimiento correspondió a las plantas sin poda con una densidad de siembra de 3,9 plantas $/ \mathrm{m}^{2}$, que obtuvo 2,76 y $2,62 \mathrm{~kg} \mathrm{~m}^{-2}$ de rendimiento total y comercial, respectivamente. En cuanto a sólidos solubles totales, a la mayor densidad se obtuvo $11,02{ }^{\circ}$ Brix, mientras que a la menor densidad el valor encontrado fue de $12,88^{\circ}$ Brix; las plantas sin poda obtuvieron mayores valores para esta variable, en comparación a las plantas podadas.

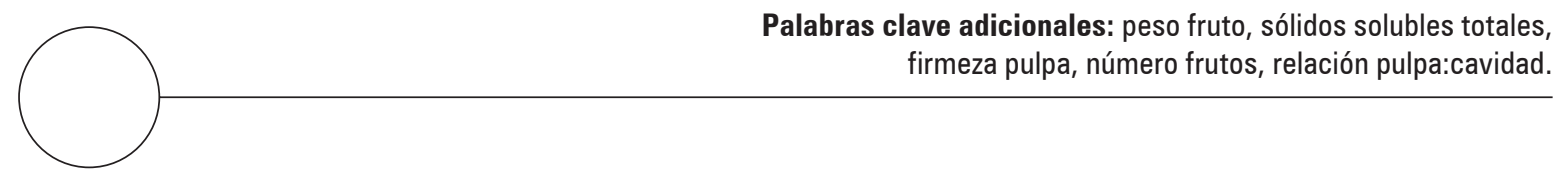

Facultad de Ciencias Agroalimentarias, Escuela de Agronomía, Universidad de Costa Rica, San José (Costa Rica).

Estación Experimental Agrícola Fabio Baudrit Moreno, Universidad de Costa Rica, San José (Costa Rica).

Autor para correspondencia.melonescr@yahoo.com.mx 


\section{ABSTRACT}

Because of a lack of information on the effect of pruning and planting density on melon grown under greenhouse conditions in Costa Rica, this study was carried out to determine the effect of three planting densities (1.9, 3.2 and 3.9 plants $/ \mathrm{m}^{2}$ ) and three pruning methods (one secondary shoot, two secondary shoots, and no pruning) on the yield and quality of Cantaloupe melon Torreón F-1. The crop was established with fertigation, using coconut fiber as the substrate. There were no significant differences between the treatments, neither for the days to start the harvest nor for the fruit pulp:cavity ratio. There were significant differences between the treatments, both for yield and for the percentage of total soluble solids. The highest yield was obtained with the highest planting density and no pruning (2.76 and $2.62 \mathrm{~kg} \mathrm{~m}^{-2}$, total and commercial yield, respectively). For the total soluble solids, $11.02{ }^{\circ} \mathrm{Brix}$ were obtained with the highest planting density, whereas $12.88^{\circ}$ Brix were measured with the lowest planting density; for this variable, the highest values were obtained in the unpruned plants, as compared to the pruned plants.

Additional key words: fruit weight, total soluble solids, pulp firmness, fruit number, pulp:cavity ratio.

Fecha de recepción: 10-01-2017 Aprobado para publicación: 15-04-2017

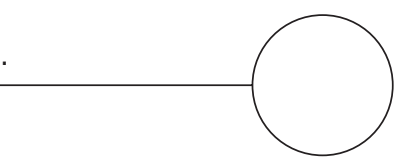

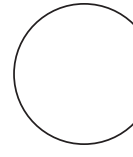

El melón (Cucumis melo L.) es uno de los cultivos económicamente más importantes en Costa Rica. Esta especie pertenece a la familia Cucurbitaceae, y presenta una planta herbácea, anual, cuyos tallos pueden ser rastreros o trepadores si se les facilita un tutorado (Reche, 2007). Los principales tipos de melón que se cultivan en este país son: Harper, Amarillo, Cantaloupe, Honey Dew, Galia, Charentais, Piel de Sapo, y Orange Flesh (Monge-Pérez, 2014). En el año 2011 el melón fue el quinto producto agrícola de mayor importancia económica, con un monto de exportaciones de 66,9 millones de dólares; el área cultivada con melón se redujo en un $50 \%$ en comparación con los años 2005-2007, debido al exceso de oferta en los mercados internacionales, la migración de la mano de obra, los efectos negativos por lluvias durante la época de cultivo, y la crisis económica de 2008 (SEPSA, 2010; SEPSA, 2012; Monge-Pérez, 2014).

El uso de ambientes protegidos es una herramienta que facilita el desarrollo de una agricultura competitiva e innovadora y permite proveer un producto de excelente calidad; en el caso de la producción de melón, permite cultivar durante todo el año, especialmente en la época lluviosa, cuando la fruta se puede vender en el mercado local a buen precio (Santos et al., 2010; Monge-Pérez, 2011). Además, se pueden alcanzar mejores rendimientos en comparación con los obtenidos a campo abierto, pues permite prolongar el ciclo productivo del cultivo (Reche, 2007; Santos et al., 2010; Alvarado-Sánchez y Monge-Pérez, 2015). Para garantizar un buen rendimiento y calidad en invernadero, se pueden utilizar tres prácticas importantes: un sistema de amarre o tutorado (cuerdas o mallas), una mayor densidad de siembra, y un sistema de podas (Gómez-Guillamón et al., 1997).

Varios investigadores han estudiado el efecto de la densidad de siembra sobre el cultivo de melón en invernadero; generalmente el rendimiento aumenta al utilizar una mayor densidad (Nerson, 1999; Gualberto et al., 2001; Pereira et al., 2003; Ban et al., 2006; Rodríguez et al., 2007; Bezerra et al., 2009; Díaz-Alvarado, 2011), y a veces esto provoca un menor peso del fruto (Nerson, 1999; Rodríguez et al., 2007), pero en otras ocasiones no se afecta el peso o la calidad del mismo (Ban et al., 2006; Rodríguez et al., 2007; Díaz-Alvarado, 2011).

Con el uso de podas lo que se busca es adelantar la cosecha, ya que en melón las flores femeninas o hermafroditas solo aparecen en las ramas secundarias o terciarias; al podar la planta se pretende mantener un balance para disminuir el vigor vegetativo y adelantar la aparición de flores femeninas o hermafroditas. Las prácticas de poda involucran la eliminación o conservación del tallo principal, y el manejo de uno, dos o más tallos secundarios y terciarios (Reche, 2007).

Varios investigadores han obtenido un mayor rendimiento al aplicar podas en melón, en comparación al testigo sin poda (Uygun y Sari, 2000; Jani y Hoxha, 2002; Pereira et al., 2003), pero en otros casos no se han presentado diferencias entre estos tratamientos 
para esta variable (Eltez et al., 1999; Uygun y Sari, 2000).

Algunos estudios han mostrado que la poda a dos tallos secundarios es la que produce el mayor rendimiento (Uygun y Sari, 2000; Jani y Hoxha, 2002; Barni et al., 2003) y el mayor peso del fruto (Barni et al., 2003), pero en otras ocasiones no se encontraron diferencias entre ese tipo de poda y otros métodos de poda, para el rendimiento, peso promedio del fruto, porcentaje de sólidos solubles totales, firmeza de la pulpa, ni la relación pulpa:cavidad del fruto (Díaz-Alvarado, 2011).

El objetivo de esta investigación fue evaluar el efecto de la combinación factorial de tres densidades de siembra y tres sistemas de poda, sobre el rendimiento y la calidad de un genotipo de melón tipo Cantaloupe, cultivado bajo condiciones de invernadero, en Alajuela, Costa Rica.

\section{MATERIALES Y MÉTODOS}

El proyecto se llevó a cabo en Barrio San José de Alajuela, Costa Rica, en el invernadero del Programa de Hortalizas de la Estación Experimental Agrícola Fabio Baudrit Moreno (EEAFBM), ubicada a $883 \mathrm{msnm}$. Se sembró el híbrido Torreón F-1, el cual es un melón tipo Cantaloupe (Cucumis melo L. var. cantalupensis), cuya planta es andromonoica (Monge-Pérez, 2016) y de crecimiento poco exuberante (J.E. Monge-Pérez, datos sin publicar).

Las plántulas fueron trasplantadas cuando tenían dos hojas verdaderas expandidas. Se utilizaron sacos plásticos de $1 \mathrm{~m}$ de largo, $20 \mathrm{~cm}$ de ancho y $15 \mathrm{~cm}$ de alto, rellenos con fibra de coco.

Se evaluaron nueve tratamientos, correspondiente a la combinación factorial de tres densidades de siembra y tres tipos de poda. La distancia entre hileras fue de 1,54 m, y la distancia entre plantas fue de 33,0; 20,0 y $16,7 \mathrm{~cm}$, correspondientes a una densidad de siembra de 1,$9 ; 3,2$ y 3,9 plantas $/ \mathrm{m}^{2}$, respectivamente. Los tipos de poda fueron: un tallo secundario; dos tallos secundarios; y plantas sin poda. La poda del tallo principal se realizó a los $15 \mathrm{~d}$ después de transplante (ddt), cuando las plantas tenían tres hojas verdaderas expandidas (Gómez-Guillamón et al., 1997), y se seleccionó uno o dos tallos secundarios, según el tratamiento correspondiente. El tratamiento sin poda se dejó a libre crecimiento.
Para todas las plantas, los tallos principales o secundarios fueron tutorados en una malla de polietileno, de 2,0 $\mathrm{m}$ de altura. En los tratamientos con poda, una vez tutorado el tallo secundario se realizaron podas semanales de la siguiente manera: los tallos terciarios que tenían fruto se podaron después de la segunda hoja emergida posterior al fruto, y los tallos terciarios sin fruto se podaron después de la cuarta hoja emergida (Gómez-Guillamón et al., 1997); esta poda de mantenimiento fue realizada durante los $22 \mathrm{~d}$ posteriores a la poda inicial.

Al inicio de la floración se introdujo una colmena de abejas (Apis mellifera) con el fin de promover una adecuada polinización.

La cosecha inició a los 75 ddt y se extendió por un período de $29 \mathrm{~d}$, hasta los $104 \mathrm{ddt}$. El índice e cosecha utilizado fue cuando había un 75\% de desprendimiento del pedúnculo, y se lograba una fácil separación del fruto.

Las variables evaluadas fueron: días a inicio de cosecha (ddt), peso promedio del fruto (g), número de frutos (totales y comerciales) por planta, rendimiento (total y comercial, en $\mathrm{kg} \mathrm{m}^{-2}$ ), porcentaje de sólidos solubles totales ('Brix), firmeza de la pulpa del fruto $\left(\mathrm{kg} \mathrm{cm}^{-2}\right)$ y relación pulpa:cavidad del fruto.

Adicionalmente se clasificaron los frutos cosechados según su peso, en rangos de $200 \mathrm{~g}$, desde un peso menor a $400 \mathrm{~g}$, hasta $1.600 \mathrm{~g}$. Se consideró como fruto comercial aquél con un peso superior a $399 \mathrm{~g}$.

Cada fruto fue evaluado individualmente, iniciando por el peso, para lo cual se utilizó una balanza electrónica marca Ocony, modelo TH-I-EK (Fa. Ocony, San José, Costa Rica), de 5.000,0 00,1 g de capacidad. Posteriormente, el fruto fue cortado longitudinalmente, y a una mitad se le evaluó el porcentaje de sólidos solubles totales, utilizando un refractómetro manual marca Atago, modelo N-1 ${ }^{a}$ (Fa. Atago, Tokio, Japón), con una capacidad de $32,0 \pm 0,2{ }^{\circ} \mathrm{Brix}$. A la otra mitad se le midió la firmeza y el grosor de la pulpa, y el grosor de la cavidad interna del fruto. Para la evaluación de la firmeza de la pulpa se usó un penetrómetro portátil marca Effegi, modelo FT-327 (Fa. Facchini, Alfonsine, Italia), con una capacidad de $12,5 \pm 0,1 \mathrm{~kg}$ $\mathrm{cm}^{-2}$, y se utilizó el puntero cuya base mide $7,5 \mathrm{~mm}$ de diámetro. Para la medición del grosor de la pulpa y de la cavidad interna del fruto se usó una cinta métrica marca Assist, modelo 32G-8025 (Fa. Ningbo Assist Tools, Yuyao, China), con una capacidad de 
$800,0 \pm 0,1 \mathrm{~cm}$. A partir de estos datos se calculó la relación pulpa:cavidad del fruto.

Se utilizó un diseño experimental irrestricto al azar, con arreglo de parcelas divididas; las parcelas grandes correspondieron a las densidades de siembra, y las pequeñas a los diferentes sistemas de podas. Se establecieron cuatro repeticiones por tratamiento. El área útil fue equivalente a $2 \mathrm{~m}$ lineales $\left(3,1 \mathrm{~m}^{2}\right)$ a lo largo de la hilera de siembra. La cantidad de plantas incluidas en cada parcela útil varió en función de la densidad de siembra: la densidad de 1,9 plantas $/ \mathrm{m}^{2}$ tuvo 6 plantas; la densidad de 3,2 plantas $/ \mathrm{m}^{2}$ tuvo 10 plantas y la densidad de 3,9 plantas $/ \mathrm{m}^{2}$ tuvo 12 plantas. Todos los frutos producidos dentro de la parcela útil fueron evaluados. Para todas las variables se realizó un análisis estadístico de varianza, y se utilizó la prueba de LSD Fisher con una significancia de 5\% para determinar diferencias entre tratamientos.

\section{RESULTADOS Y DISCUSIÓN}

No se obtuvieron diferencias significativas en los días a inicio de cosecha, entre las plantas podadas y las plantas sin poda (Tab. 1); este resultado es contrario al obtenido por Barni et al. (2003), quienes informaron que la poda a dos tallos generó un retraso en la maduración y cosecha de melón. Tampoco se presentaron diferencias para esta variable entre las diferentes densidades de siembra. En otro ensayo llevado a cabo en invernadero en la EEAFBM, el híbrido Torreón inició su cosecha a los 76 ddt, que es un resultado similar al obtenido en el presente ensayo (Monge-Pérez, 2016).

En relación al peso promedio del fruto, no se encontraron diferencias significativas entre las diferentes densidades de siembra, ni entre los distintos tipos de poda (Tab. 1). Este resultado es contrario a lo obtenido en otros ensayos con melón en invernadero, donde el número de tallos por planta sí afectó el peso promedio del fruto (Gualberto et al., 2001; Barni et al., 2003), y también es contrario a los resultados encontrados por Eltez et al. (1999), donde las plantas sin poda produjeron los frutos con mayor peso. A nivel de tratamientos, únicamente se presentaron diferencias significativas entre dos tratamientos de plantas con poda a un tallo: con la densidad de 3,2 plantas $/ \mathrm{m}^{2}$ se obtuvo un mayor peso promedio del fruto $(651,9 \mathrm{~g})$ en comparación con 3,9 plantas $/ \mathrm{m}^{2}$ (530,2 g).

En otra evaluación realizada en invernadero en la EEAFBM con el melón Torreón, el peso promedio del fruto fue de 574,3 g (Monge-Pérez, 2016), lo cual es un resultado similar a los obtenidos en el presente estudio. Sin embargo, en producción a campo abierto

Tabla 1. Días a inicio de cosecha, peso promedio del fruto, y número de frutos (totales y comerciales) por planta, para melón Cantaloupe.

\begin{tabular}{|c|c|c|c|c|c|}
\hline \multirow{2}{*}{ Efecto } & \multirow{2}{*}{ Tratamiento } & \multirow{2}{*}{$\begin{array}{l}\text { Días a inicio de } \\
\text { cosecha (ddt) }\end{array}$} & \multirow{2}{*}{$\begin{array}{l}\text { Peso promedio del } \\
\text { fruto }(\mathrm{g})\end{array}$} & \multicolumn{2}{|c|}{ Número de frutos por planta } \\
\hline & & & & Total & Comercial \\
\hline \multirow{3}{*}{ Densidad (plantas $/ \mathrm{m}^{2}$ ) } & 1,9 & $81,4 \mathrm{a}$ & $564,4 \mathrm{a}$ & $1,05 \mathrm{a}$ & $0,95 \mathrm{a}$ \\
\hline & 3,2 & $80,2 \mathrm{a}$ & $587,5 \mathrm{a}$ & $0,83 a$ & $0,71 \mathrm{a}$ \\
\hline & 3,9 & $77,3 \mathrm{a}$ & 579,3 a & $0,92 \mathrm{a}$ & $0,80 \mathrm{a}$ \\
\hline \multirow{3}{*}{ Tipo de poda } & 1 tallo secundario & $81,7 \mathrm{a}$ & $585,4 \mathrm{a}$ & $0,73 \mathrm{a}$ & $0,66 \mathrm{a}$ \\
\hline & 2 tallos secundarios & $79,9 \mathrm{a}$ & 578,6 a & $0,85 a$ & $0,75 \mathrm{a}$ \\
\hline & Sin poda & $77,4 \mathrm{a}$ & 567,3 a & $1,22 \mathrm{~b}$ & $1,05 b$ \\
\hline \multicolumn{2}{|c|}{ Interacción densidad $\times$ tipo de poda } & ns & ns & ns & ns \\
\hline \multicolumn{6}{|c|}{ Densidad $\left(\right.$ plantas $\left./ \mathrm{m}^{2}\right) \times$ tipo de poda } \\
\hline \multirow{3}{*}{1,9} & 1 tallo secundario & ns & $574,1 \mathrm{ab}$ & $0,95 a b$ & $0,89 a b$ \\
\hline & 2 tallos secundarios & ns & $564,8 a b$ & $0,96 a b$ & $0,88 a b$ \\
\hline & Sin poda & ns & $554,4 a b$ & $1,25 b$ & $1,08 \mathrm{~b}$ \\
\hline \multirow{3}{*}{3,2} & 1 tallo secundario & ns & $651,9 \mathrm{~b}$ & $0,58 \mathrm{a}$ & $0,55 \mathrm{a}$ \\
\hline & 2 tallos secundarios & ns & $550,4 a b$ & $0,73 \mathrm{a}$ & $0,63 \mathrm{a}$ \\
\hline & Sin poda & ns & $560,2 a b$ & $1,20 \mathrm{~b}$ & $0,95 a b$ \\
\hline \multirow{3}{*}{3,9} & 1 tallo secundario & ns & $530,2 \mathrm{a}$ & $0,67 \mathrm{a}$ & $0,54 \mathrm{a}$ \\
\hline & 2 tallos secundarios & ns & $620,4 \mathrm{ab}$ & $0,88 a b$ & $0,75 a b$ \\
\hline & Sin poda & ns & $587,3 a b$ & $1,21 \mathrm{~b}$ & $1,11 \mathrm{~b}$ \\
\hline
\end{tabular}

Promedios con letras distintas indican diferencia significativa según la prueba de LSD Fisher $(P \leq 0,05)$; ns: no significativa. 


Tabla 2. Distribución porcentual de la cosecha en rangos de peso del fruto, para melón Cantaloupe.
\begin{tabular}{|c|c|c|c|c|c|c|c|c|}
\hline \multicolumn{7}{|c|}{ Rango de peso de los frutos (\%) } \\
\hline $\begin{array}{c}\text { Tratamiento } \\
\text { Densidad } \\
\text { (plantas/m }{ }^{2} \text { ) }\end{array}$ & Tipo de poda & $<400 \mathrm{~g}$ & $400-600 \mathrm{~g}$ & $601-800 \mathrm{~g}$ & $801-1.000 \mathrm{~g}$ & $1.001-1.200 \mathrm{~g}$ & $1.201-1.400 \mathrm{~g}$ & $1.401-1.600 \mathrm{~g}$ \\
\hline \multirow{3}{*}{1,9} & 1 tallo secundario & 11,1 & 38,9 & 50,0 & 0 & 0 & 0 & 0 \\
\cline { 2 - 10 } & 2 tallos secundarios & 8,7 & 43,5 & 39,1 & 8,7 & 0 & 0 & 0 \\
\cline { 2 - 10 } & Sin poda & 13,3 & 50,0 & 33,3 & 3,3 & 0 & 0 & 0 \\
\hline \multirow{3}{*}{3,2} & 1 tallo secundario & 4,3 & 34,8 & 52,2 & 4,3 & 0 & 4,3 & 0 \\
\cline { 2 - 10 } & 2 tallos secundarios & 17,2 & 48,3 & 24,1 & 10,3 & 0 & 0 & 0 \\
\cline { 2 - 10 } & Sin poda & 20,8 & 37,5 & 35,4 & 6,3 & 0 & 0 & 0 \\
\hline \multirow{3}{*}{3,9} & 1 tallo secundario & 18,8 & 43,8 & 34,4 & 3,1 & 0 & 0 & 0 \\
\cline { 2 - 10 } & 2 tallos secundarios & 14,3 & 47,6 & 23,8 & 7,1 & 2,4 & 0 & 0 \\
\cline { 2 - 9 } & Sin poda & 8,6 & 46,6 & 37,9 & 6,9 & 0 & 0 & 0 \\
\hline
\end{tabular}

en Costa Rica, este híbrido produce frutos de mayor peso a los encontrados en el presente ensayo; esto se puede deber a que las zonas productoras se encuentran ubicadas a menos de $100 \mathrm{msnm}$, donde la temperatura es mayor con respecto a la que se presentó en este ensayo (J.E. Monge-Pérez, datos sin publicar).

En otro ensayo realizado en invernadero en Brasil, con poda a un tallo y 2,0 plantas $/ \mathrm{m}^{2}$, el híbrido Torreón mostró un peso promedio del fruto entre 695,5 y 998,3 g (Queiroga et al., 2008b), los que constituyen valores superiores a los obtenidos en el presente trabajo.

La cosecha se concentró principalmente en el rango de frutos entre 400 y $800 \mathrm{~g}$ para todos los tratamientos, los cuales se consideraron comerciales (Tab. 2).

En el caso del número de frutos (totales y comerciales) por planta, se observa que el tratamiento sin poda obtuvo una mayor producción que los tratamientos con poda (Tab. 1). La densidad de siembra no provocó diferencias en esta variable. En otro ensayo llevado a cabo en la EEAFBM, el melón Torreón produjo 1,88 frutos por planta (Monge-Pérez, 2016), lo que es un resultado superior al encontrado en el presente ensayo. En otros ensayos con melones Cantaloupe o reticulados en invernadero, se ha informado de una producción entre 0,40 y 4,77 frutos por planta (Pádua et al., 2003; Barni et al., 2003; Botto, 2011; Barrientos, 2013).

Con respecto al rendimiento (total y comercial), se obtuvieron mayores valores con la densidad más alta, y también con las plantas sin poda (Tab. 3). Esto es congruente con lo hallado por otros investigadores (Nerson, 1999; Gualberto et al., 2001; Pereira et al., 2003; Ban et al., 2006; Rodríguez et al., 2007; Bezerra et al., 2009; Díaz-Alvarado, 2011).
El mayor rendimiento total y comercial se obtuvo en el tratamiento de plantas sin poda y con una densidad de 3,9 plantas $/ \mathrm{m}^{2}\left(2,76\right.$ y $2,62 \mathrm{~kg} \mathrm{~m}^{-2}$, respectivamente); este rendimiento comercial es similar al rendimiento exportable obtenido en condiciones a campo abierto para este híbrido (J. E. Monge-Pérez, datos sin publicar).

En otro ensayo realizado en invernadero en la EEAFBM, a una densidad de 2,6 plantas $/ \mathrm{m}^{2}$ y $\sin$ poda, el melón Torreón presentó un rendimiento total de 2,80 kg/m² (Monge-Pérez, 2016), lo cual es similar al mayor rendimiento obtenido en la presente investigación. En un ensayo realizado en Brasil con el melón Torreón en invernadero, con poda a un tallo y 2,0 plantas $/ \mathrm{m}^{2}$, el rendimiento comercial obtenido fue de entre 1,79 y $2,83 \mathrm{~kg} / \mathrm{m}^{2}$ (Queiroga et al., 2008b); esos resultados son similares a los mayores rendimientos obtenidos en el presente ensayo.

En otros ensayos con melones Cantaloupe o reticulados en invernadero, se ha informado de un rendimiento total entre 0,27 y $5,77 \mathrm{~kg} \mathrm{~m}^{-2}$ (Pádua et al., 2003; Queiroga et al., 2008a; Morales, 2009; Bezerra et al., 2009; Charlo et al., 2009; Botto, 2011; Charlo et al., 2011; Barrientos, 2013). Sin embargo, en Rio Grande do Sul, Brasil, Barni et al. (2003) evaluaron otro genotipo de melón Cantaloupe, cultivado en el suelo en un invernadero a 2,67 plantas $/ \mathrm{m}^{2}$, y encontraron un rendimiento total entre 7,3 y $9,4 \mathrm{~kg} \mathrm{~m}^{-2}$, lo que corresponde a valores superiores a los obtenidos en el presente trabajo.

Para el porcentaje de sólidos solubles totales, se presentó una disminución en los valores obtenidos conforme aumentó la densidad de siembra (Tab. 4), lo cual es similar a lo encontrado por otros investigadores 
Tabla 3. Rendimiento (total y comercial) para melón Cantaloupe.

\begin{tabular}{|c|c|c|c|}
\hline \\
\hline Efecto & Tratamiento & Rendimiento total $\left(\mathrm{kg} \mathrm{m}^{-2}\right)$ & Rendimiento comercial $\left(\mathrm{kgm}^{-2}\right)$ \\
\hline \multirow{3}{*}{ Densidad (plantas/m²) } & 1,9 & $1,14 \mathrm{a}$ & $1,07 \mathrm{a}$ \\
\hline & 3,2 & $1,53 \mathrm{a}$ & $1,40 \mathrm{a}$ \\
\hline & 3,9 & $2,08 \mathrm{~b}$ & $1,92 \mathrm{~b}$ \\
\hline \multirow{3}{*}{ Tipo de poda } & 1 tallo secundario & $1,20 \mathrm{a}$ & $1,12 \mathrm{a}$ \\
\hline & 2 tallos secundarios & $1,47 \mathrm{a}$ & $1,36 \mathrm{a}$ \\
\hline & Sin poda & $2,08 \mathrm{~b}$ & $1,90 \mathrm{~b}$ \\
\hline \multicolumn{2}{|c|}{ Interacción densidad $\times$ tipo de poda } & ns & ns \\
\hline \multicolumn{4}{|c|}{ Densidad $\left(\right.$ plantas $\left./ \mathrm{m}^{2}\right) \times$ tipo de poda } \\
\hline \multirow{3}{*}{1,9} & 1 tallo secundario & $1,04 \mathrm{a}$ & $1,00 \mathrm{a}$ \\
\hline & 2 tallos secundarios & $1,04 \mathrm{a}$ & $1,00 \mathrm{a}$ \\
\hline & Sin poda & $1,32 \mathrm{a}$ & $1,20 \mathrm{abc}$ \\
\hline \multirow{3}{*}{3,2} & 1 tallo secundario & $1,15 \mathrm{a}$ & $1,14 a b$ \\
\hline & 2 tallos secundarios & $1,30 \mathrm{a}$ & $1,17 \mathrm{abc}$ \\
\hline & Sin poda & $2,16 \mathrm{c}$ & $1,89 \mathrm{bcd}$ \\
\hline \multirow{3}{*}{3,9} & 1 tallo secundario & $1,40 a b$ & $1,23 \mathrm{abc}$ \\
\hline & 2 tallos secundarios & $2,08 \mathrm{bc}$ & $1,91 \mathrm{~cd}$ \\
\hline & Sin poda & $2,76 \mathrm{c}$ & $2,62 \mathrm{~d}$ \\
\hline
\end{tabular}

Promedios con letras distintas indican diferencia significativa según la prueba de LSD Fisher $(P \leq 0,05)$; ns: no significativa.

Tabla 4. Componentes de calidad interna de fruto, para melón Cantaloupe.

\begin{tabular}{|c|c|c|c|c|}
\hline Efecto & Tratamiento & $\begin{array}{c}\text { Sólidos solubles totales } \\
\left(\%{ }^{\circ} \text { Brix }\right)\end{array}$ & $\begin{array}{c}\text { Firmeza de la pulpa } \\
\left(\mathrm{kg} \mathrm{cm}^{-2}\right)\end{array}$ & Relación pulpa:cavidad \\
\hline \multirow{3}{*}{ Densidad (plantas $/ \mathrm{m}^{2}$ ) } & 1,9 & $12,88 \mathrm{c}$ & $2,81 \mathrm{a}$ & $1,25 \mathrm{a}$ \\
\hline & 3,2 & $11,93 \mathrm{~b}$ & $2,94 \mathrm{a}$ & $1,19 \mathrm{a}$ \\
\hline & 3,9 & $11,02 \mathrm{a}$ & $2,91 \mathrm{a}$ & $1,19 \mathrm{a}$ \\
\hline \multirow{3}{*}{ Tipo de poda } & 1 tallo secundario & $11,78 \mathrm{a}$ & $2,96 \mathrm{a}$ & $1,19 \mathrm{a}$ \\
\hline & 2 tallos secundarios & $11,60 \mathrm{a}$ & $2,90 \mathrm{a}$ & $1,22 \mathrm{a}$ \\
\hline & Sin poda & $12,44 \mathrm{~b}$ & $2,80 \mathrm{a}$ & $1,23 \mathrm{a}$ \\
\hline \multicolumn{2}{|c|}{ Interacción densidad $\times$ tipo de poda } & ns & $\mathrm{ns}$ & ns \\
\hline \multicolumn{5}{|c|}{ Densidad (plantas $\left./ \mathrm{m}^{2}\right) \times$ tipo de poda } \\
\hline \multirow{3}{*}{1,9} & 1 tallo secundario & $13,00 \mathrm{c}$ & $2,93 a b$ & ns \\
\hline & 2 tallos secundarios & $12,58 \mathrm{c}$ & $2,90 a b$ & ns \\
\hline & Sin poda & $13,05 \mathrm{c}$ & $2,60 \mathrm{a}$ & ns \\
\hline \multirow{3}{*}{3,2} & 1 tallo secundario & $11,45 \mathrm{~b}$ & $2,95 a b$ & ns \\
\hline & 2 tallos secundarios & $11,68 \mathrm{~b}$ & $2,83 \mathrm{ab}$ & ns \\
\hline & Sin poda & $12,68 \mathrm{c}$ & $3,05 \mathrm{~b}$ & ns \\
\hline \multirow{3}{*}{3,9} & 1 tallo secundario & $10,90 a b$ & $3,00 \mathrm{ab}$ & $\mathrm{ns}$ \\
\hline & 2 tallos secundarios & $10,55 \mathrm{a}$ & $2,98 \mathrm{ab}$ & ns \\
\hline & Sin poda & $11,60 \mathrm{~b}$ & $2,75 a b$ & ns \\
\hline
\end{tabular}

Promedios con letras distintas indican diferencia significativa según la prueba de LSD Fisher $(P \leq 0,05)$; ns: no significativa.

(Nerson, 2002; García et al., 2009), aunque otros autores no encontraron diferencias significativas para esta variable al evaluar diferentes densidades de siembra (Rodríguez et al., 2007; Díaz-Alvarado, 2011). También se observó una disminución de los valores de esta variable en los tratamientos con poda, con respecto a las plantas sin poda. En otro ensayo en Brasil, se determinó que la poda a dos tallos produjo frutos con un mayor porcentaje de sólidos solubles totales, en relación a la poda a un tallo (Gualberto et al., 2001), pero en el presente estudio no se encontraron diferencias significativas entre esos tratamientos. 
Los valores obtenidos para el porcentaje de sólidos solubles totales son similares a los informados para el híbrido Torreón en otro ensayo en invernadero en la EEAFBM, donde obtuvo 13,0 ${ }^{\circ}$ Brix (Monge-Pérez, 2016), y en un ensayo en invernadero en Brasil, donde obtuvo entre 11,4 y 12,1 ${ }^{\circ}$ Brix (Queiroga et al., 2008b). Todos los valores obtenidos para esta variable en el presente estudio están por encima de 10,0 ${ }^{\circ} \mathrm{Brix}$, que es el mínimo requerido para que el fruto de melón se considere comercial y sea aceptado en la mayoría de los mercados (Sáenz, 2005). En otros ensayos en invernadero con melones Cantaloupe o reticulados, se ha informado de valores para esta característica entre 6,10 y $14,06^{\circ}$ Brix (Rizzo y Braz, 2001; Castoldi et al., 2008; Vargas et al., 2008; Queiroga et al., 2008a; Charlo et al., 2009; Morales, 2009; Botto, 2011; Charlo et al., 2011; Barrientos, 2013).

Con respecto a la firmeza de la pulpa, no se presentaron diferencias entre las diferentes densidades de siembra, ni entre los distintos tipos de poda. A nivel de tratamientos, únicamente se encontraron diferencias significativas entre dos tratamientos de plantas sin poda: con la densidad de 3,2 plantas $/ \mathrm{m}^{2}$ se presentó una mayor firmeza de pulpa $\left(3,05 \mathrm{~kg} \mathrm{~cm}^{-2}\right)$ en comparación con 1,9 plantas $/ \mathrm{m}^{2}\left(2,60 \mathrm{~kg} \mathrm{~cm}^{-2}\right)$. Esta variable está ligada a la capacidad del fruto para mantenerse adecuadamente durante el período poscosecha, aunque también es importante para obtener un fruto crujiente, característica deseada por algunos mercados. A nivel de fincas exportadoras de melón en Costa Rica, se ha definido un valor mínimo aceptable de firmeza de pulpa de $2,0 \mathrm{~kg} \mathrm{~cm}^{-2}$ (Monge-Pérez, 2011), por lo que se concluye que todos los tratamientos tienen una firmeza de pulpa apropiada.

No se encontraron diferencias significativas para la relación pulpa:cavidad del fruto entre los diferentes tratamientos de densidad de siembra y de tipos de poda. Todos los tratamientos obtuvieron para esta variable valores superiores a 1 , por lo que los frutos se consideran de buena calidad.

\section{CONCLUSIONES}

La densidad de siembra afectó el rendimiento (total y comercial) y el porcentaje de sólidos solubles totales en el melón Cantaloupe cultivado en invernadero.

El tipo de poda afectó el número de frutos por planta (totales y comerciales), el rendimiento (total y comercial), y el porcentaje de sólidos solubles totales en el melón Cantaloupe cultivado en invernadero.

El mayor rendimiento (total y comercial) correspondió a las plantas sin poda, con una densidad de siembra de 3,9 plantas $/ \mathrm{m}^{2}$.

Con respecto al porcentaje de sólidos solubles totales, a la mayor densidad de siembra se obtuvo $11,02^{\circ}$ Brix, mientras que a la menor densidad el valor encontrado fue de $12,88^{\circ}$ Brix. Las plantas sin poda obtuvieron mayores valores para esta variable, en comparación a las plantas podadas.

\section{AGRADECIMIENTOS}

Los autores agradecen el financiamiento recibido por parte de la Vicerrectoría de Investigación de la Universidad de Costa Rica para la realización de este trabajo. Asimismo, agradecen la colaboración de Cristina Arguedas, Julio Vega, Carlos González y Andrés Oviedo en el trabajo de campo, y de Mario Monge en la revisión de la traducción del resumen al idioma inglés.

\section{REFERENCIAS BIBLIOGRÁFICAS}

Alvarado-Sánchez, T. y J.E. Monge-Pérez. 2015. Efecto de la aplicación de bioactivadores y del raleo manual de frutos sobre el rendimiento y la calidad de melón (Cucumis melo L.) bajo cultivo protegido en Costa Rica. Tecnol. Marcha 28(4), 15-25. Doi: 10.18845/tm.v28i4.2439

Ban, D., S. Goreta y J. Borosic. 2006. Plant spacing and cultivar affect melon growth and yield components. Sci. Hortic. 109, 238-243. Doi: 10.1016/j. scienta.2006.04.015

Barni, V., N.A. Barni y J.R. Silveira. 2003. Meloeiro em estufa: duas hastes é o melhor sistema de condução. Ciência Rural 33(6), 1039-1043. Doi: 10.1590/ S0103-84782003000600007

Barrientos, M.A. 2013. Cultivo protegido hidropónico de melón (Cucumis melo L.) en la zona norte de Costa Rica. Práctica de especialidad. Escuela de Agronomía, sede Regional de San Carlos, Instituto Tecnológico de Costa Rica, Cartago, Costa Rica.

Bezerra, F.M.L., M.C.H. Nunes, C.A.S. Freitas y F.L. Silva. 2009. Desempenho de três híbridos de meloeiro sob dois espaçamentos em ambiente protegido na Chapada do Apodi. Rev. Ciênc. Agron. 40(3), 412-416.

Botto, A.S. 2011. Evaluación del rendimiento y el total de sacarosa disuelta $\left({ }^{\circ} \mathrm{Bx}\right)$ de quince cultivares de melón (Cucumis melo L.) en sustrato compost y mezcla compost con arena bajo condiciones de macrotúnel. 
Proyecto especial. Escuela Agrícola Panamericana, Zamorano, Honduras.

Castoldi, R., H.C.O. Charlo, P.F. Vargas y L.T. Braz. 2008. Qualidade de frutos de cinco híbridos de melão rendilhado em função do número de frutos por planta. Rev. Bras. Frutic. 30(2), 455-458. Doi: 10.1590/ S0100-29452008000200032

Charlo, H.C.O., R. Castoldi, P.F. Vargas y L.T. Braz. 2009. Desempenho de híbridos de melão-rendilhado cultivados em substrato. Cient. 37(1), 16-21.

Charlo, H.C.O., F.S. Galatti, L.T. Braz y J.C. Barbosa. 2011. Híbridos experimentais de melão rendilhado cultivados em solo e substrato. Rev. Bras. Frutic. 33(1), 144156. Doi: 10.1590/S0100-29452011005000028

Díaz-Alvarado, J. 2011. Efecto del tipo de poda y la densidad de plantas sobre el rendimiento y la calidad en frutos de melón Amarillo (Cucumis melo L.) cultivados en un sistema sin suelo, bajo condiciones de invernadero. En: Taller de producción de melón en invernadero. 15-07-2011. Estación Experimental Agrícola Fabio Baudrit Moreno, Universidad de Costa Rica, San José, Costa Rica.

Eltez, R.Z., Y. Tüzel y K. Boztok. 1999. Effects of different growing media and pruning methods on greenhouse muskmelon production. Acta Hortic. 491, 363-368. Doi: 10.17660/ActaHortic.1999.491.56

García, J.C., G. Rodríguez, J.G. Lugo y V. Rodríguez. 2009. Efecto del cultivar y distancia entre plantas sobre características físico-químicas del fruto del melón ( $\mathrm{Cucu-}$ mis melo L.). Rev. Fac. Agron. (LUZ) 26, 141-158.

Gómez-Guillamón, M.L., R. Camero y J. Gonzáles-Fernández. 1997. El melón en invernadero. pp. 67-77. En: Namesny, A. (ed.). Melones. Compendios de horticultura. No. 10. Ed. Horticultura, Barcelona, España.

Gualberto, R., F.V. Resende y P.H.L. Losasso. 2001. Produtividade e qualidade do melão rendilhado em ambiente protegido, em função do espaçamento e sistema de condução. Hortic. Bras. 19(3), 240-243. Doi: 10.1590/ S0102-05362001000300018

Jani, S. y S. Hoxha. 2002. The effect of plant pruning on production of melon grown under PVC greenhouse conditions. Acta Hortic. 579, 377-381. Doi: 10.17660/ ActaHortic.2002.579.65

Monge-Pérez, J.E. 2016. Evaluación de 70 genotipos de melón (Cucumis melo L.) cultivados bajo invernadero en Costa Rica. InterSedes 17(36), 1-41. Doi: 10.15517/ isucr.v17i36.26944

Monge-Pérez, J. E. 2014. Producción y exportación de melón (Cucumis melo) en Costa Rica. Tecnol. Marcha 27(1), 93-103. Doi: 10.18845/tm.v27i1.1700

Monge-Pérez, J.E. 2011. Aspectos económicos en la producción de melón. En: Taller de producción de melón en invernadero. 15-07-2011. Estación Experimental Agrícola Fabio Baudrit Moreno, Universidad de Costa Rica, San José, Costa Rica.
Morales, F. 2009. Caracterización de producción de genotipos de melón reticulado (Cucumis melo L.) bajo invernadero 2008-2009. Trabajo de grado. División de Carreras Agronómicas, Universidad Autónoma Agraria "Antonio Narro", Unidad Laguna, Torreón, Coahuila, México.

Nerson, H. 2002. Relationship between plant density and fruit and seed production in muskmelon. J. Amer. Soc. Hortic. Sci. 127(5), 855-859.

Nerson, H. 1999. Effects of population density on fruit and seed production in muskmelons. Acta Hortic. 492, 6570. Doi: 10.17660/ActaHortic.1999.492.7

Pádua, J.G., L.T. Braz, D.A. Banzatto, M.T.A. Gusmão y S.A.L. Gusmão. 2003. Net melon cultivars productivity under different cultivation systems, during summer and winter. Acta Hortic. 607, 83-89. Doi: 10.17660/ ActaHortic.2003.607.12

Pereira, F.H., I. Nogueira, J. Pedrosa, M. Negreiros y F. Bezerra-Neto. 2003. Poda da haste principal e densidade de cultivo na produção e qualidade de frutos em híbridos de melão. Hortic. Bras. 21(2), 191-196. Doi: 10.1590/ S0102-05362003000200014

Queiroga, R.C.F., M. Puiatti, P.C.R. Fontes y P. R. Cecon. 2008a. Produtividade e qualidade de frutos de meloeiro variando número de frutos e de folhas por planta. Hortic. Bras. 26(2), 209-215. Doi: 10.1590/ S0102-05362008000200016

Queiroga, R.C.F., M. Puiatti, P.C.R. Fontes y P.R. Cecon. 2008b. Produtividade e qualidade do melão cantaloupe, cultivado em ambiente protegido, variando o número e a posição dos frutos na planta. Bragantia 67(4), 911-920. Doi: 10.1590/S0006-87052008000400013

Reche, J. 2007. Cultivo intensivo del melón. Hojas divulgadoras No. 2125 HD. Ministerio de Agricultura, Pesca y Alimentación, Madrid, España.

Rizzo, A.A.N. y L.T. Braz. 2001. Características de cultivares de melão rendilhado cultivadas em casa de vegetacão. Hortic. Bras. 19(3), 370-373. Doi: 10.1590/ S0102-05362001000300017

Rodríguez, J.C., N.L. Shaw y D.J. Cantliffe. 2007. Influence of plant density on yield and fruit quality of greenhouse-grown galia muskmelons. Hort Technol. 17(4), 580-585.

Sáenz, M.V. 2005. Biología y fisiología de los productos frescos. pp. 64-90. En: Meléndez, G. y G. Umaña (eds.). Memoria del curso de capacitación "Sistemas poscosecha en frutas de mango, melón y sandía: conceptos y aplicaciones". Instituto Nacional de Innovación y Transferencia en Tecnología Agropecuaria (INTA), San José, Costa Rica.

Santos, B., H. Obregón-Olivas y T. Salamé-Donoso. 2010. Producción de hortalizas en ambientes protegidos: estructuras para la agricultura protegida. Publicación 
HS1182. IFAS Extension, UF Department of Horticultural Sciences, University of Florida, Gainesville, FL.

SEPSA. 2012. Boletín estadístico agropecuario No. 22, Serie cronológica 2008-2011. Secretaría Ejecutiva de Planificación Sectorial Agropecuaria, San José, Costa Rica.

SEPSA. 2010. Boletín estadístico agropecuario No. 20, serie cronológica 2006-2009. Secretaría Ejecutiva de Planificación Sectorial Agropecuaria, San José, Costa Rica.
Uygun, N. y N. Sari. 2000. The effects of different pruning methods and height of fruit setting on plant growth, yield and fruit quality of melons grown in greenhouses. Turk. J. Agric. For. 24, 365-373.

Vargas, P.F., R. Castoldi, H.C.O. Charlo y L.T. Braz. 2008. Qualidade de melão rendilhado (Cucumis melo L.) em função do sistema de cultivo. Ciênc. Agrotecn. 32(1), 137-142. Doi: 10.1590/S1413-70542008000100020 